\title{
A proposed routing scheme for power reduction in wireless networks
}

\author{
Dalia I. Elewely *, Marwa F. Areed , Hesham A. Ali \\ Computer engineering and systems department, faculty of engineering, Mansoura university, Mansoura, Egypt \\ *Corresponding author E-mail: dalia.el3wely@gmial.com
}

Copyright $\odot 2014$ Dalia I. Elewely et al. This is an open access article distributed under the Creative Commons Attribution License, which permits unrestricted use, distribution, and reproduction in any medium, provided the original work is properly cited.

\begin{abstract}
Ad-hoc networks consist of a set of mobile nodes with a restricted power supply resources that can communicate with each other without any established infrastructure or centralized administration. The loss of some nodes may cause significant topological changes, undermine the network operation, and affect the lifetime of the network. This paper discusses the energy consumption problem and summaries the existing power saving techniques in ad-hoc wireless networks. The main objective of this paper is to introduce a new power aware multi-path node disjoint routing scheme based on the Dynamic Source Routing protocol (DSR), which can prolong MANET's lifetime, reduce routing delay and increase the reliability of the packets reaching its destination. Therefore, a comprehensive study of DSR protocol has been drawn using NS-2 simulator, to evaluate the performance of DSR as a routing strategy and investigate its efficiency in saving wireless networks resources, as a prelude to avoid its performance shortcomings in our proposed routing scheme.
\end{abstract}

Keywords: Power aware protocol, node disjoint, network simulation 2, multipath routing, Dsr protocol, ad-hoc network.

\section{Introduction}

Ad-hoc network is an infrastructure-less dynamic mobile network that is formed automatically as a result of the connections between a set of mobile nodes that are located in a specific area. In this kind of networks, nodes are the main component and each node can function as a router which discover and maintain routes to other nodes in the network, as well, as an end-device (transmitter and receiver). The main characteristics of the Ad-hoc networks are dynamic topology, band-width constrained, variable capacity links and energy constrained operation, due to these characteristics, the path between two nodes may be unstable and prone to be broken. Routing or find the optimal path between the source and destination nodes in Ad-hoc networks is the most fundamental research issue.

As depicted in Fig. (1); current routing protocols of Ad-hoc networks can be classified depending on their network structure or routing strategy. Based on the network structure, routing protocols can be classified into "flat routing", "hierarchical routing" and "geographic position assisted routing" [1], while according to routing strategy, routing protocols can be classified into "table driven" also called "proactive", "on-demand" also called "reactive" and "hybrid" which combines the advantages of both proactive and reactive routing [2].

On-demand and table driven routing protocols, are considered as flat routing protocols [1], where all nodes participate in the routing process equally. Table driven routing attempts to maintain information about the path from each node to every other node in the network, and it works well in networks that have low node mobility or frequently transmission of data. Example of protocols that use this routing strategy; "Destination Sequenced Distance Vector" DSDV [3] and Optimized Link State Routing (OLSR) [4]. On-demand routing protocols were designed as the routes between two nodes are created only when source has packets to be sent to its destination and doesn't have a route to reach it, in this case a discovery process is initiated to find the shortest available path. Ad hoc on-demand Distance Vector (AODV) [5], Dynamic Source Routing (DSR) [6] and Temporally Ordered Routing Algorithm (TORA) protocols use this strategy. As the size of network increases the flat routing may cause too much overhead in the network, so hierarchal routing will be suitable in this case, such as "Zone Routing Protocol" ZRP [7] (hybrid routing protocol) and "Hierarchal State 
Routing" HSR [1], that have different solutions to the organization of routing of nodes. Geographic position assisted routing also called geographical routing, is based on the idea that the source sends a message to the geographic location of the destination instead of using the network address [1].this kind of routing prevent network-wide researches for destination, however the routing updates must be done faster than the network mobility rate to make an effective location based routing. "Distance Routing Effect Algorithm for Mobility "DREAM and "Greedy Perimeter Stateless Routing" GPSR considered as geographical protocols.

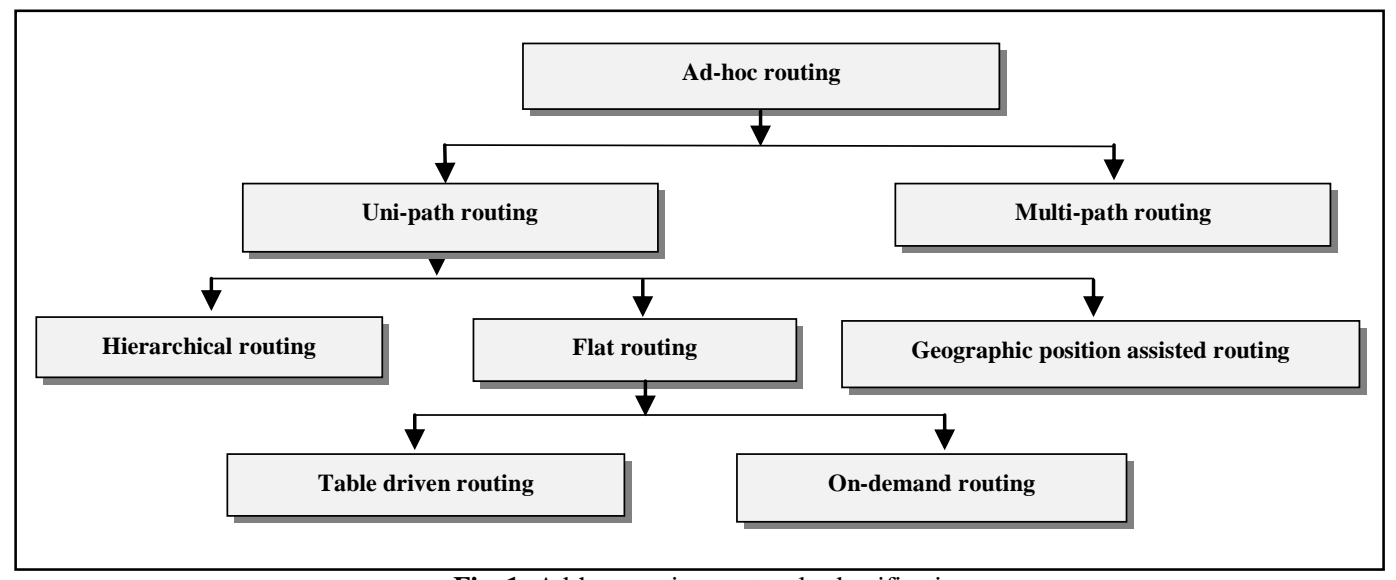

Fig. 1: Ad-hoc routing protocols classifications.

All these previous protocols, considered as uni-path protocols, i.e. one route is established and maintained between source and destination pairs to deliver specific message. Otherwise, multi-path routing protocols that establish multiple routes between the source node and its destination. Based on previous uni-path routing protocols, many multi-path protocols are proposed, Ad-hoc on-demand Multipath Distance Vector (AOMDV) [8], AODV backup routing (AODV-BR) [9], Split Multi-path Routing (SMR) [10] and Multi-path Source Routing (MSR) [11], are considered as multi path protocols. The AOMVD and AODV-BR were based on the AODV routing protocol, whereas SMR and MSR were based on DSR. Although uni-path routing have a lower overhead of route discovery than Multi-path one, Multipath routing have many benefits over uni-path, it provides load balancing, fault-tolerance, minimum end to end delay, higher overall throughput and lower frequency of the route discovery initiation process.

\subsection{Dynamic source routing protocol}

DSR [6] is an on-demand source routing protocol that was developed at Carnegie Mellon University in 1996. It composed of two main mechanisms; route discovery and route maintenance that work together to allow nodes to discover and maintain routes to destinations. Source routing means that source must know the complete hop sequence to its destination. On-demand means that route discovery and route maintenance are initiated only if a source does not have a route to its destination or detects a broken route. Also, DSR requires no periodic exchange of packets at any layer within the network (unlike AODV in which each node sends hello packets to its neighbors periodically). Accordingly, overhead that caused by periodic activity is reduced to zero. Each node also maintains a route cache in which it caches source routes that it has learned recently.

\subsection{Ad-hoc on-demand distance vector routing protocol}

AODV [7] is a variation of DSDV routing protocol which is collectively based on DSDV and DSR. In AODV protocol, as same as protocols that use the on-demand routing strategy, it does not maintain routes from every node to every other node in the network. Route discovery mechanism is initiated only when a node wants to send a data packet to its destination, each node also have a routing table in which it stores routes that it has been learned recently.

The rest of the paper is organized as follows: in the next section we will summarize the power consumption problem in ad-hoc networks and the existing power aware protocols; in section 3, the performance of DSR protocol will be evaluated using ns2; in section 4, related work will be listed, and our new routing scheme will be presented; conclusion is in section 5

\section{Problem formula \& existing solutions}

In ad hoc networks, nodes are usually power constrained as they depend on limited battery resources that usually consumed through the communication between these nodes. As ad-hoc networks are based on multi-hop communication, energy of intermediate nodes is also spend by forwarding packets for other nodes. Many ad-hoc 
Routing protocols are designed in a way that the optimal path is the path with the minimum number of hops from source to destination (i.e. the shortest path), thus some nodes become involved in routing packets for many source destination pairs. Over time, the battery of these nodes will get depleted and this will cause node failure and therefore path failure. This failure may lead to a network partition. In order to achieve the objective of maintaining the connection between network nodes for as long as possible (i.e. maximize the network lifetime), the distribution of tasks or load among its nodes should be balanced so that they all decrease power in the same rate and run out of energy at the same time.

Through the last few years a number of power reduction researches have been proposed, these researches have targeted different layers of the network protocol stack. However most such researches has been targeted at MAC layer and the network layer by developing different power aware protocols at these two layers. As depicted in Fig. (2); Existing power aware routing protocol algorithms can be classified into activity-based which can find a power conservation solutions that related to the transmission of data between nodes in the network i.e. network activity, and connectivitybased control that its aim is to find solution that related to transmission connectivity maintenance for an ad-hoc network [12]. Connectivity-based protocols can be divided into two categories, Topology control and Passive energy saving protocols. Topology control protocol, such as Local Information No Topology (LINT) [13] and Local Information LinkState Topology (LILT) [13], in these protocols, each node adjusts its transmission signal according to the number of (dh) and (dl) neighbors. LINT uses only local information, i.e. it can't recognize network partitions. Passive energy saving, such as Geographic Adaptive Fidelity (GAF) [14], where the ad-hoc network is divided into virtual grids, each grid must keep enough nodes in active state to ensure the network connectivity, and place other nodes within its grid into a sleeping state to conserve energy.

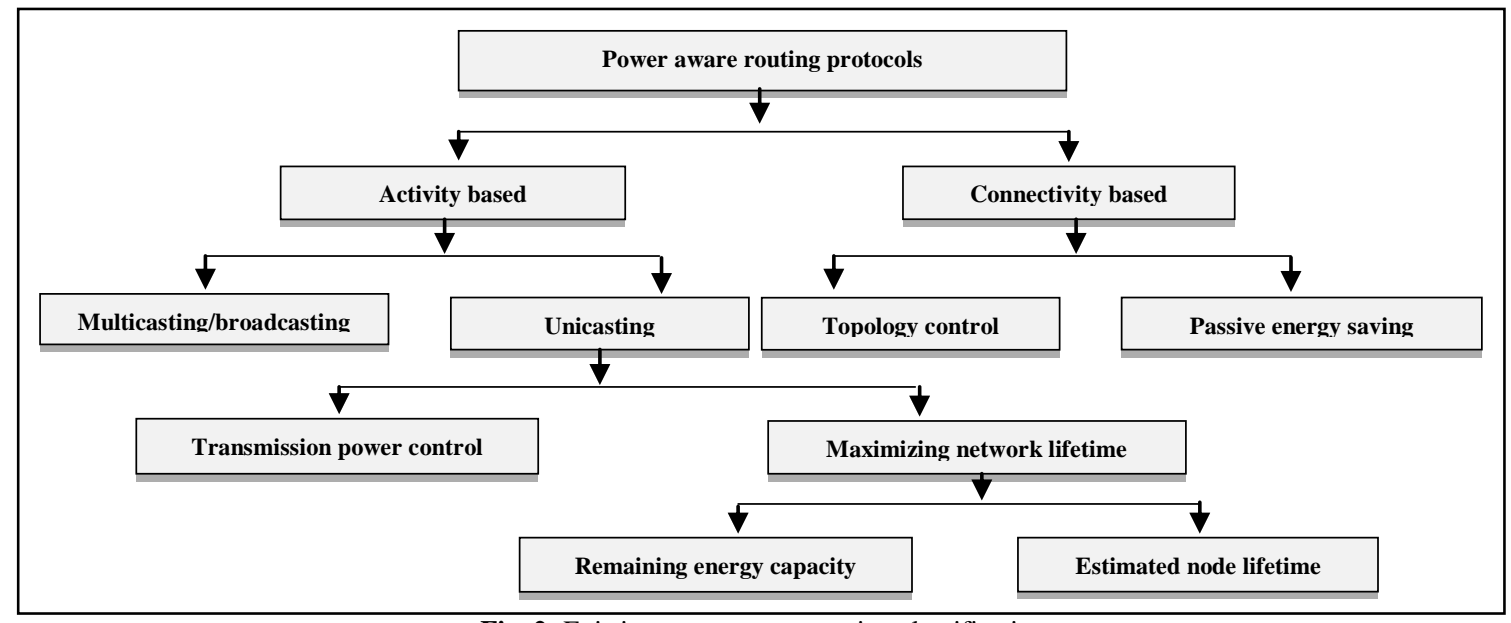

Fig. 2: Existing power aware routing classification.

Activity-based protocols can be classified into transmission power control and maximizing network lifetime protocols. Transmission power control protocols use the transmission power as a metric, the Minimum Total Transmission Power Routing (MTPR) [15] and Minimum Power Routing protocol (MPR) [16] belong to this category which its goal is to select the path with minimum energy consumption. Maximizing network lifetime protocols utilize the ad-hoc network power resources with a balanced manner to prolong its lifetime. Lifetime maximization protocols can be classified according to their path selection metric into two categories, "remaining energy capacity" which uses the residual energy as a cost metric and "estimated node lifetime". In [17] the authors proposed Min-sum Battery Cost Routing (MBCR) protocol that chooses the optimal path with the minimum cost i.e. with the maximum residual energy, where the path cost is the summation of the remaining energies of nodes that form this path. One drawback of this protocol that a path with a large number of nodes all has a low level of remaining energy may be selected as an optimal path! Thus path failure may occur. Min-Max Battery Cost routing protocol (MMBC) [17] overcomes MBCR problem, it selects the path with the minimum cost and with the highest minimum remaining energy capacity node than other paths. Conditional Min-Max Battery Cost Routing protocol (CMMBCR) [17] is a hybrid of the min-sum and the min-max battery cost routing algorithms, When the battery capacity of some nodes goes below a predefined threshold routes going through these nodes will be avoided, and therefore the time until the first node power-down is extended. Local Energy Aware Routing (LEAR) has been proposed in [18], in this protocol, when the remaining energy in a node is lower than a predefined threshold level, it does not participate in the route discovery phase. Thus avoid network overhead and energy consumption.

Although remaining energy capacity cost metric is used by many power aware routing protocols, this cost does not grantee the lifetime of a node, because a node with high energy capacity can also get depleted if there is high traffic load passing through it. in $[19,20]$, authors proposed the drain rate metric that is defined as the rate at which energy gets dissipated at a given node, The drain rate is computed by an exponential weighted moving average method and gives the estimated energy dissipation per second. Lifetime Prediction Routing (LPR) has been proposed in [21], where each 
node tries to estimate its battery lifetime based on its past activity. This is achieved using a Simple Moving Average (SMA) predictor.

Most researchers evaluated and compared the performance of the new power aware routing protocol with its non-power aware one. C-K. Toh in [22] evaluated the performance of MBCR, MMBCR and CMMBCR, the number of expired nodes using MBCR became smaller compared to use MMBCR as a routing protocol, CMMBCR showed better results compared to the other two protocols depending on how the threshold value was chosen. Authors in [19] compared MDR with MMBCR algorithm. The results showed that Minimum Drain Rate (MDR) [20] avoids over-dissipation however it does not guarantee that the total transmission power is minimized over a chosen route as in MMBCR. Repeating the simulation with different scenarios in [23] showed that MTPR can find the minimum energy cost path and conserve energy compared to MMBCR, MDR and CMMBCR. However, MMBCR and MDR can more evenly distribute energy consumption among nodes in the network, thereby extend the network lifetime.

\section{Performance analysis of DSR protocol}

Many papers have been presented to evaluate and compare the performance of on-demand routing protocols DSR and AODV and table driven protocol DSDV, using different simulators such as OMNeT++, Opnet, GloMoSim, MATLAB and NS2. Authors in [24] presented a comprehensive analysis of DSR protocol performance using ns2 simulator with varying simulation pause time and traffic load, the study showed that DSR has a better performance with high pause time and high number of connections. In [25] simulation has been done varying the speed of the nodes with a constant pause time, then varying the pause time with a constant speed, the comparison between DSR, AODV and DSDV was based on Average End-to-End Delay, Routing Load and Packet Delivery Fraction an a performance metrics, as expected DSDV has the highest PDF, AODV has the lowest average end-to-end delay. In [26] the author made a performance comparison between DSR and DSDV varying Pause time, the result indicated that DSR has a superior performance than DSDV especially in scenarios with law pause time. Authors in [27] made a comparison between DSR and AODV using PDF and Normalized load as a performance metrics, varying pause time, they repeated the simulation many times with a different number of sources, from 10 to 40, results showed that AODV has a higher PDF than DSR, while DSR has a lower normalized routing load.

\subsection{Performance metrics}

There is a number of performance metrics that can be used to study the performance of on-demand routing protocols, such as:

a) Packet Delivery Ratio (PDF): is the ratio of number of packets received at the destination to the number of packets sent from the source, i.e. fraction of successfully received packets, which survive while finding their destination.

b) Average End-to-End Delay (AEED): Average time delay for data packets from the source node to its destination node.

c) Average Throughput: is the average number of successful packets that deliver in certain time to their destinations over a communication channel.

d) Normalized Routing Load (NRL): is defined as the total number of routing packet transmitted per data packet.

e) Energy Consumption Ratio: is the ratio of average consumed energy of all nodes at the end of the simulation.

\subsection{Simulation environment}

In our evaluation of DSR and AODV protocols performance, NS-2.34 is used as a simulation tool under Windows 7 operating system using Cygwin as a Linux emulation tool. NS-2 is a discrete event object-oriented network simulator that has been widely used in networks research area [28].The simulation scripts are written using OTCL language, mobility scenarios are generated using "setdest" tool and traffic scenarios are generated using "cbrgen.tcl" tool. "AWK" scripts are used to read and analyze the output trace files.

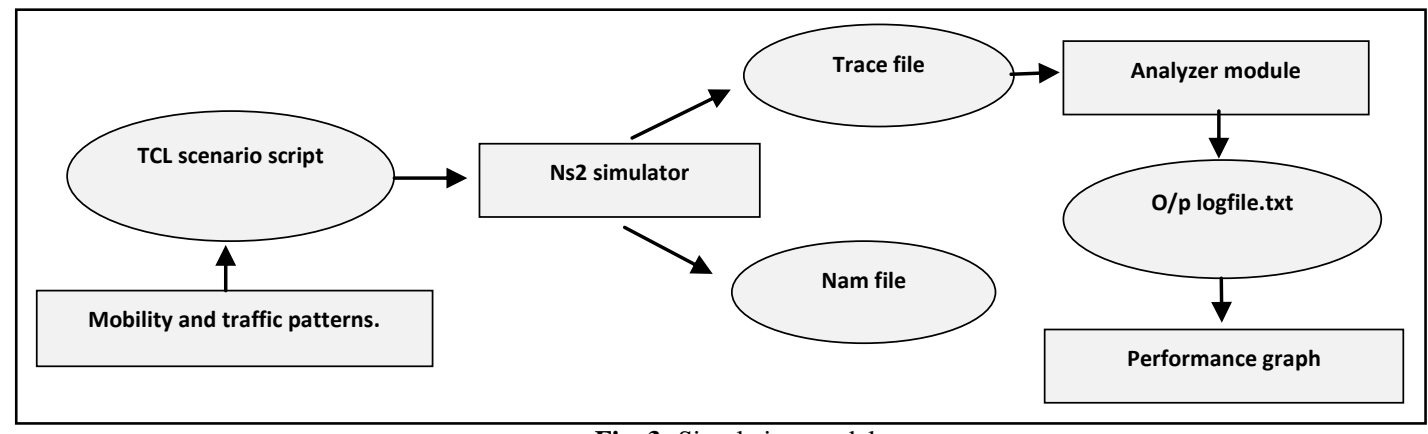

Fig. 3: Simulation model. 
In the simulation environment, the number of mobile nodes are varying (from 10 to 50) with a 250m propagation radius, randomly distributed in a 500m X 500m region, nodes move according to the widely used random waypoint Model, In this model, each node begins the simulation by remaining stationary for 50 seconds as a pause time . It then selects a random destination in the simulation area and moves to that destination at a speed distributed uniformly between 0 and 10 meter per second. Upon reaching the destination, the node pauses again for 50 seconds, selects another destination, and proceeds there as previously described, repeating this behavior for the duration of the simulation which is 200 seconds. The traffic is generated by 10 TCP sources. The sending rate was set to 4 packets per second, and the data packet's size was set to 512 bytes. The Distributed Coordination Function (DCF) of IEEE 802.11 [29] for wireless LANs is used as the MAC layer protocol. NS-2 energy model is used with 100 Jules as an initial energy for each node, all nodes are assumed to have the same amount of battery capacity with full energy at the beginning of the simulation. Simulation parameters are appended in Table 1.

Table 1: Simulation parameters

\begin{tabular}{llll}
\hline \multicolumn{4}{c}{ Table 1: Simulation parameters } \\
\hline Parameter & Value & Parameter & Value \\
\hline Simulator & NS-2.34 & Traffic type & TCP \\
Simulation time & $200 \mathrm{~s}$ & Packet size & 512 bytes \\
Simulation area & $500 \times 500 \mathrm{~m}^{2}$ & Queue type & Drop tail \\
No. of nodes & $5-50$ nodes & Propagation model & TwoRayGround \\
Routing protocol & DSR and AODV & Transmission range & $250 \mathrm{~m}$ \\
MAC layer & IEEE 802.11 & Initial energy & $100 \mathrm{Joules}$ \\
Mobility model & Random waypoint & Transmission power & $0.7 \mathrm{~W}$ \\
Pause time & $50 \mathrm{~s}$ & Receiving power & $0.3 \mathrm{~W}$ \\
No. of data sources & 10 sources & Idle Power & $0.1 \mathrm{~W}$ \\
\hline
\end{tabular}

\subsection{Performance results}

The simulation results are in the form of line graphs that indicate the impact of increasing the number of simulated mobile nodes on the network performance metrics. Graphs in Fig.4 show a comparison between DSR and AODV routing protocols on the basis of (PDF, AEED, Avg. throughput and NRL), respectively, as a functions of the number of mobile nodes, using average TCP traffic load.

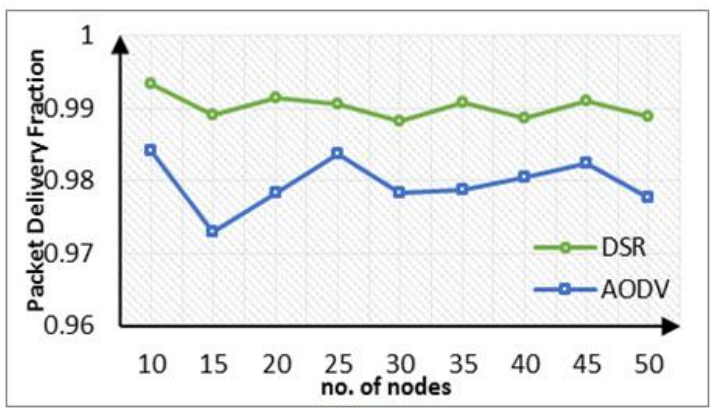

(a)

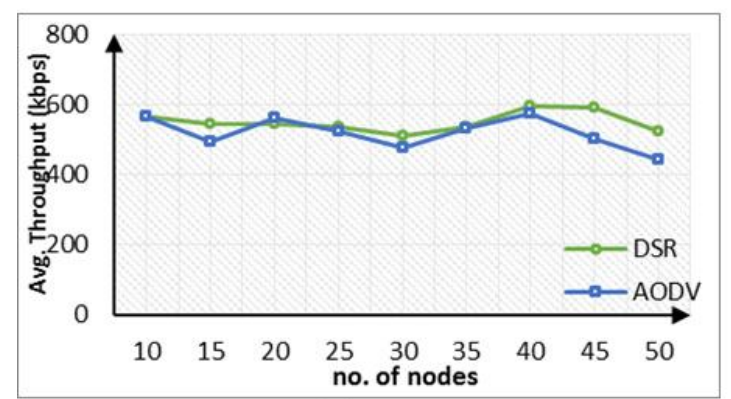

(c)

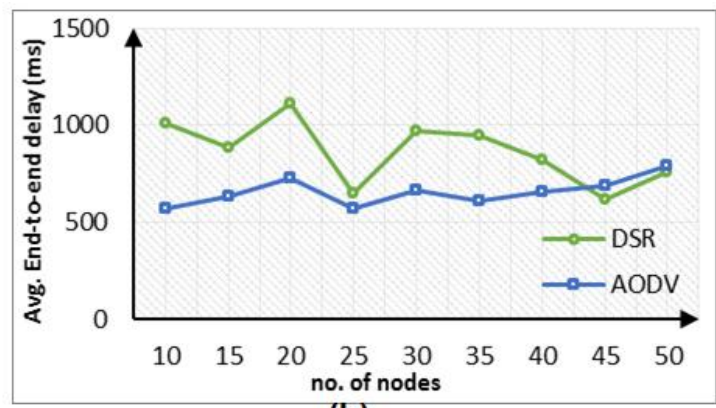

(b)

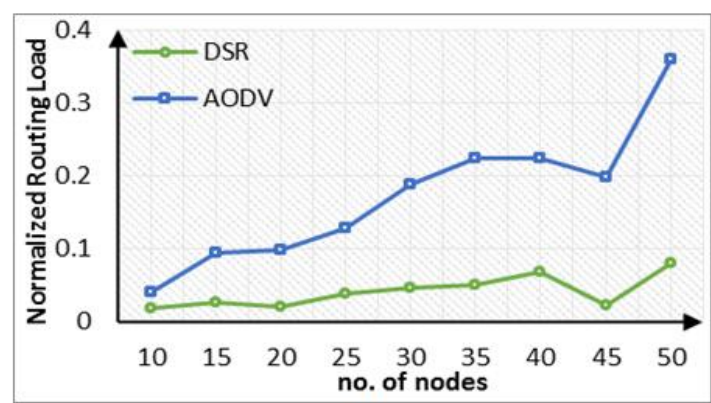

(d)

Fig. 4: Impact of the network size.

Fig. 4(a) shows that the ratio of packet delivery of both protocols is above 97\%, PDF is higher in DSR than AODV; as DSR source nodes use fresh routes from their local caches, and this would provide more reliability in packet delivery. Observed that there is no impact of the density of nodes in the network on the PDF values of both protocols. Fig. 4(b) 
indicates that data packets that routed using AODV have a little delay time until reaching their destination, compared to packets that routed using DSR protocol. There is a clear difference between them. This is because in DSR protocol, intermediate node spends a lot of time processing control packets that it received. Observed that at " 25 nodes" scenario and at high number of node (40 and 45), DSR and AODV registered almost convergent AEED values. Fig. 4(c) shows that DSR and AODV almost have a close Avg. Throughput values, DSR registers better values with the network size growth. As mentioned before, the most distinguished feature of DSR as a routing technique that there are no periodic messages exchanges, and this leads to control packets overhead reduction, so as expected, in Fig. 4(d), even with a TCP traffic load; "NRL" values of DSR is very low compared to the values of AODV that consumes network bandwidth in the periodic beaconing.

From the previous analysis, DSR registered superior performance with low density networks, but in general, DSR under normal or average conditions has a higher performance compared to AODV protocol. These results led us to make a power consumption evaluation to DSR, as a prelude to turn it into power-aware multi-path protocol.

\subsection{Energy consumption analysis of DSR protocol}

In our energy consumption analysis, same parameters from subsection (3.2) is used, except simulation time, pause time and traffic load. Simulation time is extended to 400 s to test the effect of long time simulation on the energy consumption in networks using DSR protocol. Simulation is repeated 10 times, with constant number of nodes (25 nodes); as DSR showed a moderate performance results in networks that consist of that number of nodes. Through the first five times, we run the simulation under light TCP traffic load using 10 TCP connections between 5 sources and their destinations, then we repeated the simulation under heavy TCP traffic load using 40 connections between 20 sources and their destinations for 5 other times. For each five times, pause time value changed from 0s to 400 s with average speed between $3.91 \mathrm{~m} / \mathrm{s}$ and $0.56 \mathrm{~m} / \mathrm{s}$. the change in these selected parameters is to study the effect of the dynamicity and the velocity on the network power resources with low and heavy traffic loads.

Fig. 5(a) Shows the total percentage of energy consumed due to the sending and the receiving for all packet types: TCP (Data packets), DSR (control packets), RTS (MAC packets) and ARP packets. It is observed that in all cases of varying pause time with traffic load, energy consumption is higher during receiving process (above 50\%). The receiving process includes actual receipt of data and overhearing data from neighbor nodes.

Fig. 5(b) shows the percentage of energy consumed based on all kinds of these packets. It observed that MAC packets have a major effect on energy consumption. These comparisons show that networks with high pause time and low traffic load register better results than those with low pause time with high traffic loads. The more dynamicity of the network increases, the more initiation of new route discovery processes occurs, and this leads to more energy consumption. Despite this, network with 100 pause time and 5 sources considered as an exception. Fig. 5(c) shows line graph that compares between high and low traffic loads on the basis of PDF as a function of pause time variation. As expected, low traffic loads register high results (above 99\%), this result shows that even in high mobility or dynamicity networks, sources can send its packets effectively to their destinations. Unlike High traffic loads that register poor results (below 93\%) especially with low pause time networks. From Fig. 5(b \& c) observed that, in case of high dynamicity networks, whether with high or low traffic loads (due to quick topological changes); high packet delivery ratio can be translated into high number of route discovery processes that consume the network's power resources during finding the optimal path and processing control packets. Because of the nature of the DSR protocol as a routing technique, each node has to handle all control packets it receives even if it is not the intended recipient.

The number of expired nodes at the end of simulation time is showed in Fig. 5(d) as a function of pause time. It is clear that networks with low traffic loads have a low number of expired nodes (equal or below 5 nodes), except in case of high pause time. In static networks, source nodes always use the same path that is the shortest path to route data packets to its destination, thus, the energy of intermediate nodes that form this path (even with low traffic loads that continue for a long period of time) will be depleted quickly during forwarding process. The high dynamicity of the network decreases the number of expired nodes, but in the same time does not grantee the efficiency of the packet delivery. High traffic loads networks register high number of expired nodes, more than the half of the network nodes expired before 10 seconds of the simulation end time, observed that most of the expired nodes are source nodes.

In general, networks that uses DSR as a routing mechanism consumes less energy with low traffic loads and medium pause time. Varying pause time consumes more energy with high loads and low loads depending on the causes. This result led us to propose the following power aware routing scheme that can adapt to the network changes and activities, extend network's nodes operational life, avoid network partition and prolong networks lifetime. 


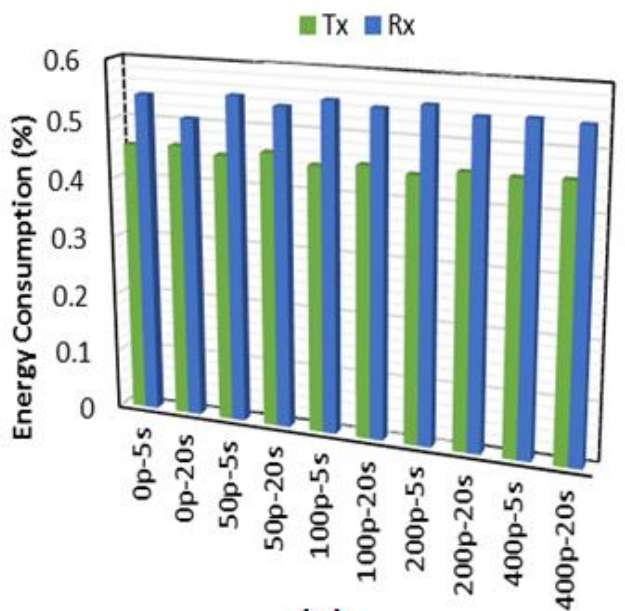

(a)

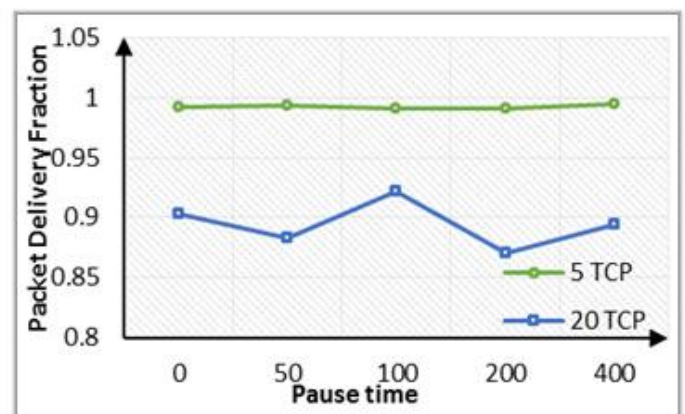

(c)

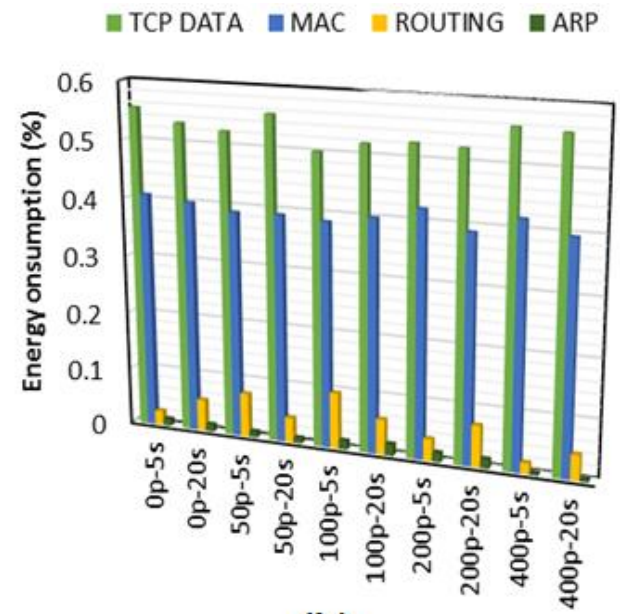

(b)

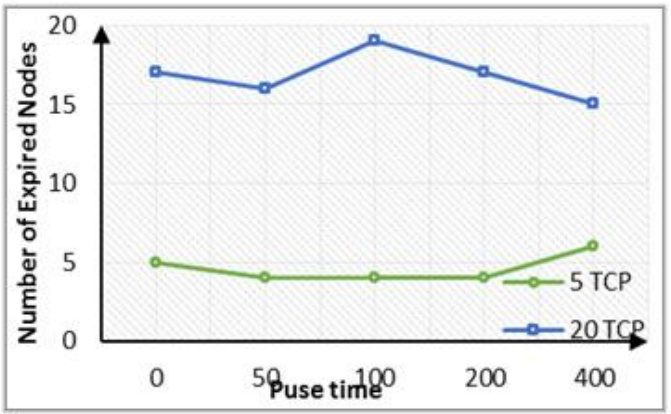

(d)

Fig. 5: Impact of the Pause time increase.

\section{Proposed power aware multipath node disjoint routing scheme}

Recently many researches have presented a variety of power aware multipath routing schemes based on on-demand protocols, as mentioned before, in on-demand routing strategy, the initiation of route discovery process is occurred only when the source node needs to route data packets to specific destination and does not have any cached route to it. From subsection (3.4) we can conclude that the on-demand mechanism saves energy and bandwidth resources that can be wasted through the periodic exchange of information that occurs in other routing strategies. Authors in [30] presented the Energy Aware Source Routing (EASR) that acts as EAR protocol [34] in saving nodes energy but with enhanced delay aspect. EASR discovers and selects paths that are not overlapped, and also uses overhearing ratio to control the overhearing energy waste. The multi-path Energy aware DSR protocol (MEA-DSR) [31] integrates the minimum drain rate metric in the multipath DSR, also a new cache update and round robin scheduling have been implemented to improve the energy and the traffic load balancing. L. Tan, L. Xie, K. Ko, M. Lei and M. Zukerman proposed the Lifetime-Aware Multipath optimized Routing (LAMOR) [32], the routing algorithm is optimized in terms of the lifetime of paths, which are essential for discovering strongly reliable routes. LAMOR supports high-speed real time video transmission in WANETs. Power-Aware Multi-Path Routing Protocol for a Wireless Ad Hoc Network (PAMP) [33] is designed as an extension of the existing AODV by modifying RREQ and RREP management mechanism to handle energy reservation and multiple paths.

Our proposed Power Aware Multipath node disjoint routing scheme depends on three mechanisms that integrate with each other through the routing discovery phase to establish multiple powers and load aware reliable disjoint connections that can extend the network's nodes operational life and maximize the network lifetime. These mechanisms are:

1) Power and load aware composite metric: A lifetime value will be calculated for each node that participates in the route discovery processes. Node lifetime value is calculated using an equation that involves, (a) the remaining energy in this node, (b) its initial energy at the beginning of the simulation, (c) the traffic loads that pass through it and (d) its transmission signal power. According to this value the optimal paths will be selected. The optimal path is a path with high lifetime nodes values and with higher minimum life time value.

2) Multi-path routing: Our proposed scheme depends on selecting and establishing two optimal routes between the source and its destination, that (as mentioned in section 1) will grantee the reliability of connections and will increase the fault tolerance. Source nodes always have alternative paths to their destination nodes, and this will reduce the frequency of initiating route discovery processes, thereby energy consumption in route discovery process will be 
reduced. Multiple paths also achieve load balancing by spreading the traffic along the two paths, and this will reduce congestion and bottlenecks, thus energy consumption will be distributed fairly among the network nodes.

3) Node disjoint paths: Although establishing multiple paths between source and destination pairs has many benefits over uni-paths, multiple paths may intersect in one or more common nodes. Although the traffic is separated among these paths, these common nodes will get depleted faster as the traffic load on these node will be much higher than the other nodes of the paths, and this leads to multiple paths failure at the same time, thereby more route discovery processes will be initiated and more resources will be consumed. Besides, with the increase of the dynamicity of the network, the probability of path failure increases, these common nodes may move away from its one hop neighbor's transmission range at any time due its mobility nature. To avoid his problem, the node disjointness between the two optimal paths will be granted at the selection paths process, i.e. two optimal paths that don't have any common nodes except the source and the destination will be selected. This will protect the network from the partitioning problem and save more resources.

This proposed routing scheme is based on on-demand source routing protocol DSR. Modifications will be done at the route discovery and route maintenance phases of DSR. All this modification will be done based on the implementation of DSR protocol in ns2 simulator. Fig. (6) Shows a general perception of the modified Route discovery phase. Most important modifications are clarified in the following six points:

1) In Route Request (RREQ) packet, two more fields will be added to its source header. The first field is PLT which will carry the path lifetime value. When an RREQ packet passes through a node, its lifetime value will be calculated and will be added to this field. At the destination node (after adding its lifetime value), this field will contain the total summation of the lifetime values of all nodes that forms the path that RREQ passed through from the source to the destination. Second field is MLT, when an RREQ packet passed through a node, if its lifetime value is lower than the RREQ's MLT value, this field will be updated by the node by copying its lifetime value, and otherwise, this field won't be updated.

2) Each node in DSR protocol has a table called Route Request table, when an RREQ packet passed through a node, some information from the RREQ fields is stored into its table. The structure of this table will be modified, three columns will be added, the first column for PLT values, the second one for MLT values and the RREQ's path field will be stored in the Path column.

3) In DSR protocol when an intermediate node receives a duplicate RREQ packet (whose source address and ID are same as Source address and ID of currently received one), intermediate node drops it. In our proposed scheme, some comparisons between this Duplicate RREQ and the original RREQ will be done. The MLT and PLT values of the duplicate RREQ will be compared with the original one. The information of the one with the highest values will be stored in the route request table and the other one will be dropped, if both packets have the same values, then the comparison will be done based on the hop count value.

4) A superior difference between this presented scheme and DSR is that intermediate nodes are not allowed to reply to the source request with routes from their caches, destination node only have the right to send route reply packets (RREP) to the source node, in order to avoid the conflict with the node disjointness mechanism.

5) The selection of the two optimal disjoint paths will be done at the destination node, and then two RREP packets will be sent from the destination to its source, each one through its optimal path, source node will store them into its cache and the transmission of data packets will begin.

6) The source node will not initiate a route discovery process until it receives the second Request Error (RERR) packet that informs it about the failure of the second path, in this case, the source node will erase this expired route from its local cache and another route discovery process will be initiated to continue the data transmission. It is expected that this proposed routing scheme will register better performance compared to DSR protocol. Even with the increase of traffic loads in high dynamicity networks, due to the power and load aware multipath node disjoint mechanisms, the connection between the source node and its destination will be prolonged as long as possible. It is supposed that this scheme may increase the overhead during the route discovery processes, due to the increase of control packet size and time that intermediate nodes will take in processing control packets. But in return, the frequency of initiating route discovery processes will be reduced, and the network lifetime will be prolonged.

\section{Conclusion \& future work}

In this paper, the energy consumption problem in Ad-Hoc networks has been discussed, and the existing power aware routing algorithms and their classifications have been summarized. A performance comparison between DSR and AODV protocols has been done using ns2 as a simulation tool. The simulation results revealed that DSR acts more efficiently than AODV does under average conditions, and DSR is more convenient as a routing strategy with small size networks. An energy consumption analysis of DSR protocol has been evaluated, results showed that, varying pause time and traffic loads have a major effect on DSR performance, high traffic loads consume the network resources and minimize its lifetime, the probability of initiating route discovery processes increases with the decreases of pause time, and this also consumes the network resources and affect its lifetime. Based on these results a new power aware multipath node disjoint routing scheme has been proposed. A new composite metric has been drawn as an effective way 
to distinguish between paths to select the two optimal ones. The Multipath node disjoint mechanism will be integrated with the power and load aware metric to distribute the energy consumption among all network nodes in balanced manner as a way to extend the operational life of the nodes and maximize the network lifetime. It is believed that this routing scheme will overcome the drawbacks and the performance shortcomings of DSR protocol.

The next step is to implement this proposed routing scheme in ns2, and then evaluate its performance compared to different power aware protocols.

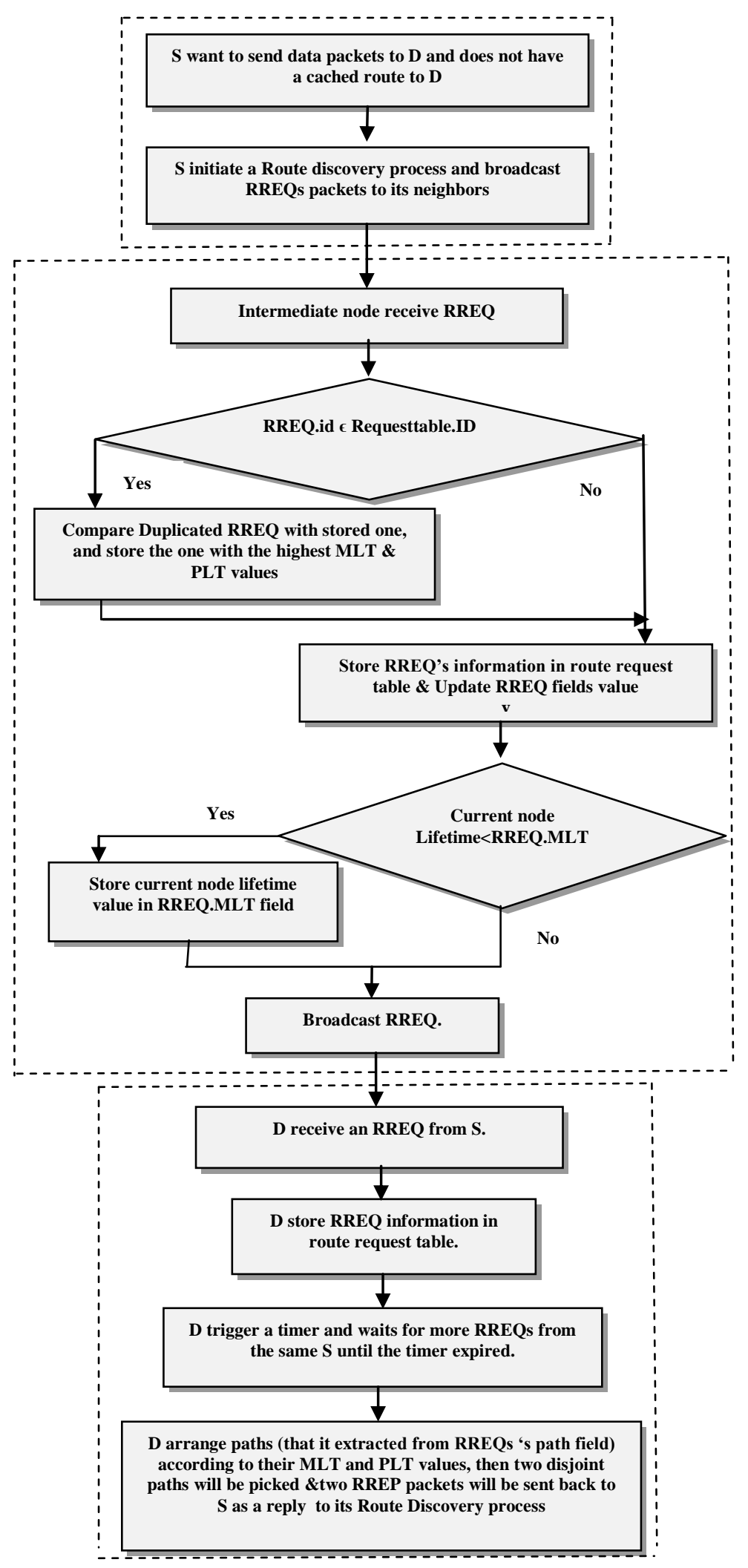

Fig. 6: Modified Route Discovery Phase. 


\section{References}

[1] X. Hong, K. Xu, and M. Gerla,"Scalable Routing Protocols for Mobile Ad Hoc Networks", In Proceedings of IEEE Network, pages 11 -21, August 2002.

[2] E. M. Royer and C. Toh, "A review of current routing protocols for ad hoc mobile wireless networks. Technical report, University of California and Georgia Institute of Technology, USA, 1999.

[3] C. E. Perkins and P. Bhagwat, "Highly dynamic Destination-Sequenced Distance-Vector routing (DSDV) for mobile computers", In Proceedings of the SIGCOMM '94 Conference on Communications Architectures, Protocols and Applications, pages 234-244, August 1994.

[4] P. Jacquet, P. Muhlethaler, T. Clausen, A. Laouiti, A. Qayyum, and L. Viennot, "Optimized link state routing protocol for ad hoc networks", In Proceedings of IEEE Multi Topic Conference (INMIC 2001), Pakistan, pages 62-68, December 2001.

[5] C. E. Perkins and E. M. Royer, "Ad-hoc On-Demand Distance Vector Routing", In PROCEEDINGS OF THE 2ND IEEE WORKSHOP ON MOBILE COMPUTING SYSTEMS AND APPLICATIONS, 1997.

[6] D. B. Johnson and D. A. Maltz, "Dynamic source routing in ad hoc wireless networks", 1996.

[7] Z.J Haas, "A new routing protocol for the reconfigurable wireless networks", In Proceedings of the 6th IEEE International Conference on Universal Personal Communications (ICUPC'97), volume 2, San Diego, pages 562-566, CA, USA, October 1997.

[8] M. K. Marina and S. R. Das, "On-demand Multipath Distance Vector Routing in Ad Hoc Networks", In Proceedings of IEEE International Conference on Network Protocols (ICNP), November 2001.

[9] S. Lee and M. Gerla, "AODV-BR: backup routing in ad hoc networks", In Proceedings of Wireless Communications and Networking Conference, 2000. WCNC. 2000 IEEE, Volume: 3, Chicago, IL, pages 1311 - 1316, September 2000.

[10] S. Lee and M. Gerla, "Split multipath routing with maximally disjoint paths in ad hoc networks", In Proceedings of Communications, 2001. ICC 2001. IEEE International Conference, Volume10, Helsinki, pages 3201 - 3205, Jun. 2001.

[11] L. Wang, Y. Shu, M Dong and L Zhang, "Adaptive multipath source routing in ad hoc networks", In Proceedings of Communications, 2001. ICC 2001. IEEE International Conference on (Volume: 3), Helsinki, pages 867 - 871, Jun. 2001.

[12] J. Sriniva , C. Reddy,” Enhancement Study of Power-Aware Routing Protocols in Ad hoc Networks", International Journal of Advanced Research in Technology Vol. 1 Issue 1, pages 71-75, Sep. 2011.

[13] R. Ramanathan and R. Rosales-Hain, "Topology control of multihop wireless networks using transmit power adjustment," In Proceedings of INFOCOM 2000. Nineteenth Annual Joint Conference of the IEEE Computer and Communications, Tel Aviv, Pages 404 - 413, 2000.

[14] J. Gomez and A.T. Campbell, “ Variable-Range Transmission Power Control in Wireless Ad Hoc Networks", IEEE Transactions on Mobile Computing, vol.6(1), Pages 87-99, January 2007.

[15] K. Scott and N. Bambos,"Routing and channel assignment for low power transmission in PCS," ICUPC '96, Cambridge, MA, 1996.

[16] M. Subbarao, "Dynamic power-conscious routing for MANETs: an initial approach," in 50th IEEE Vehicular Technology Conference (VTC’99), vol. 2, Amsterdam, The Netherlands, Pages 1232-1237, Sep. 1999.

[17] C.K. Toh, "Maximum Battery Life Routing to Support Ubiquitous Mobile Computing in Wireless Ad hoc Networks", IEEE Communication Magazine, Pages 138-147,June 2001.

[18] S.-M. Senouci and M. Naimi, "New routing for balanced energy consumption in mobile ad hoc networks," in PE-WASUN '05: Proceedings of the 2nd ACM international workshop on Performance evaluation of wireless ad hoc, sensor, and ubiquitous networks, New York, NY, USA: ACM Press, Pages 238-24, 2005.

[19] D. Kim, J.J. Garcia-Luna-Aceves, K. Obraczka, J-C. Cano, and P. Manzoni, " Routing Mechanisms for Mobile Ad Hoc Networks based on the Energy Drain Rate," IEEE Transactions on Mobile Computing, Vol. 2, No. 2, Pages. 161-173, April-June 2003.

[20] D. Kim, J.J. Garcia-Luna-Aceves, K. Obraczka, J-C. Cano, and P. Manzoni, "Power-Aware Routing Based on the Energy Drain Rate for Mobile Ad Hoc Networks," Proc. IEEE ICCCN2002: International Conference on Computer Communication and Networks, Miami, Florida, October 14-16, 2002.

[21] M. Maleki, K. Dantu, and M. Pedram, "Lifetime prediction routing in mobile ad hoc networks," in IEEE Wireless Communications and Networking, 2003. WCNC 2003, vol. 2, New Orleans, Pages 1185- 1190, Mar 2003,.

[22] C-K. Toh, Hiroshi Cobb, and David A. Scott, "Performance evaluation of battery life aware routing scheme for wireless ad hoc networks" IEEE, Pages 2824-2829, 2001.

[23] L. Cao, T. Dahlberg and Y. Wang, "Performance Evaluation of Energy Efficient Ad Hoc Routing Protocols", Pages 306-313, 2007.

[24] S. Kat and K Kumar, "Performance Analysis Of Dynamic Source Routing Protocol In wireless Mobile Ad Hoc Network", International Journal of Engineering Reserch \& Tecnology, Vol. 1, December 2012.

[25] Khandakar, Amith, "Step by Step Procedural Comparison of DSR, AODV and DSDV Routing protocol", International Proceedings of Computer Science \& Information Tech;2012, Vol. 40, Page 36, June 2012.

[26] B. Rath, "Implementing and Comparing DSR and DSDV Routing Protocols for Mobile Ad Hoc Networking", 2009.

[27] S. Shah, A. Khandre, M. Shirole and G. Bhole, "Performance Evaluation of Ad Hoc Routing Protocols Using NS2 Simulation", Mobile and Pervasive Computing (CoMPC-2008), 2008.

[28] The Network Simulator - ns-2, Website: "http://www.isi.edu/nsnam/ns/".

[29] IEEE Computer Society LAN MAN Standards Committee. Wireless LAN Medium Access Control (MAC) and Physical Layer (PHY) Specifications, IEEE Standard 802.11-1997. The Institute of Electrical and Electronics Engineers, New York 1997.

[30] Do-Youn H, Eui-Hyeok K, and Jae-Sung L, "An Energy Aware Source Routing with Disjoint Multipath Selection for Energy Efficient Multihop Wireless Ad hoc Networks", in Proceedings of International Federation for Information Processing, Pages 41-50, 2006.

[31] F. De Rango, P. Lonetti, and S. Marano, "MEA-DSR: A Multipath Energy-aware Routing Protocol for Wireless Ad Hoc Networks", IFIP International Federation for Information Processing Volume 265, pages 215-225, 2008.

[32] T. Liansheng, X. Ling, T. K. King, M. Lei and M. Zukerman, "LAMOR: Lifetime-Aware Multipath Optimized Routing Algorithm for Video Transmission over Ad hoc Networks", in Proceedings of IEEE Vehicular Technology Conference, Vol. 2, pages 623-627, 2006. Jin S. Y, K. Kang, Y. J. Cho and S. Y. Chae, "Power-Aware Multi-Path Routing Protocol for Wireless Ad hoc Network", in Proceedings of IEEE Wireless Communications and Networking Conference, Pages 2247-2252, 2008.

[33] R. C. Shah and J. M. Rabaey, "Energy Aware Routing for Low Energy Ad Hoc Sensor Networks", IEEE Wireless Communication and Networking Conference, 2002. 